\title{
SUBGINGIVAL IRRIGATIONS WITH POVIDONE- IODINE AS ADJUNCTIVE TREATMENT OF CHRONIC PERIODONTITIS
}

\author{
K. Kotsilkov, D. Emilov, Chr. Popova \\ Department of Periodontology, \\ Faculty of Dental Medicine, Medical University-Sofia. Bulgaria
}

\section{ABSTRACT:}

BACKGROUND: Successful prevention and treatment of periodontitis is contingent upon effective control of the periodontopathic microbiota. Periodontal pathogens reside in deep subgingival sites but also colonize supragingival plaque, tongue dorsum and other oral sites. Antimicrobial agents administered systemically or locally can help suppress periodontal pathogens in periodontal sites and in the entire mouth.

MATERIALS AND METODS: Thirty patients with generalized severe chronic periodontitis with $\mathbf{5 6 7}$ periodontal pockets $\mathbf{5} \mathbf{5 m m}$ were included in the presented study. The mechanical periodontal treatment was performed with ultrasonic device P5 Newtron in four visits. Subgingival irrigation of the periodontal pockets in the right two quadrants (split-mouth study) were performed with $10 \%$ povidone-iodine solutions. Full mouth periodontal status evaluation was recorded again on the last visit.

RESULTS: The results from this study show significant improvement of the clinical parameters of periodontitis in all treated patients and in almost all periodontal sites. In the regions treated with subgingival irrigation with $10 \%$ povidone-iodine solution superior reduction of the probing depth, more attachment gain and better reduction of the gingival inflammation were recorded in comparison with regions treated with SRP only.

CONCLUSION: This study demonstrates positive effect of the subgingival irrigations with $10 \%$ povidoneiodine in cases of severe chronic periodontitis. This results are rationale for future studies on the effectiveness of the iodophore solutions in the treatment of the disease and on the clinical significance of the investigated treatment.

Key words: chronic periodontitis, adjunctive antimicrobial therapy, povidone-iodine.

\section{INTRODUCTION:}

Periodontitis is a disease attributable to multiple infectious agents and exhibiting complex cellular and humoral immune responses. Much remains to be learned about the etiopathogenesis and effective management of the disease. Successful prevention and treatment of periodontitis is contingent upon effective control of the periodontopathic microbiota. Periodontal pathogens reside in deep subgingival sites but also colonize supragingival plaque, tongue dorsum and other oral sites. Controlling destructive periodontal disease warrants a comprehensive antimicrobial approach that targets periodontal pathogens in various ecological niches of the oral cavity. Scaling and root planing, with or without periodontal surgery, along with proper oral hygiene, constitute the primary approach to controlling periodontopathogens. Antimicrobial agents administered systemically or locally can help suppress periodontal pathogens in periodontal sites and in the entire mouth $(5,11)$.

Elemental iodine or its derivatives [polyvinylpyrrolidone-iodine complex (PVP-iodine)] are probably the most broad-spectrum and potent antiseptics available. Dilute PVP-iodine may be able to kill Aggregatibacter actinomycetemcomitans, Porphyromonas gingivalis and other periodontal pathogens in vitro in as little as $15 \mathrm{~s}$ of contact and bacteria and yeasts in vivo within $5 \mathrm{~min}$ of contact $(2,3,4,6,9)$.

PVP iodine is also effective against herpesviruses, which show resistance to chlorhexidine, which is of interest because of the suspected significance of cytomegalovirus and Epstein-Barr virus in destructive periodontal disease $(7,10)$.

Alongside the classical indications for PVP-iodine application in medicine, such as the disinfection of the skin and hands, mucosa antisepsis and wound treatment, recent studies have pointed to the usefulness of PVP-iodine rinsing of body cavities and joints and application to the eye. Short or long-term exposure to PVP-iodine does not induce bacterial resistance and resistance to antibiotics does not influence the sensitivity of bacteria to PVP-iodine. Allergic sensitization to PVP iodine is rare, only $0.73 \%$ of 600 patients showed a sensitization reaction in a skin patch test. With respect to toxicity, there are no reports of impaired wound healing of skin or mucosa and no untoward systemic or tissue reactions after intraoral use of PVP-iodine $(5,8)$.

However, PVP-iodine should not be administered to individuals who are allergic to iodine, suffering from thyroid dysfunction, or are pregnant or nursing $(5,11)$. 
PVP-iodine can kill periodontal bacteria and decrease postoperative bacteraemia following oral surgery (1).

The American Dental Association and the American Heart Association have suggested using PVP-iodine for subgingival irrigation but relatively few scientific data exist on its utility in periodontal treatment. Considering its potent and broad-spectrum antimicrobial activity, good safety profile and low financial cost, it seems reasonable to propose the use of PVP-iodine in the disinfection of periodontal lesions.

\section{GOAL:}

The goal of the presented study is to evaluate the effectiveness of the subgingival irrigations with $10 \%$ povidone-iodine solution as an adjunctive treatment to the basic mechanical periodontal therapy of patients with severe chronic periodontitis.

\section{MATHERIALS AND METHODS:}

Thirty patients with generalized severe chronic periodontitis with 567 periodontal pockets e" $5 \mathrm{~mm}$ were included in the presented study. One periodontal pocket of $5 \mathrm{~mm}$ per quadrant was the minimal inclusion criteria. Patients with periodontal or systemic antibiotic treatment in the last 6 months, allergic to iodine, hyper/hypotireoid or smokers were excluded from the study. The mechanical periodontal treatment was performed with ultrasonic device P5 Newtron in four visits. On the first visit full mouth periodontal status (HI, PBI, PD and CAL) was recorded along with supragingival debridement (tip N1 and abrasive aerosol). The following three visits (once per week) included removal of the supragingival plaque (abrasive aerosol) and subgingival ultrasonic scaling (tip H3-frontal sextant, tips H4L/H4R-distal sextants). Subgingival irrigation of the periodontal pockets in the right two quadrants (split-mouth study) were performed with $10 \%$ povidone-iodine solutions (3 irrigations with $1 \mathrm{ml}$ solution with interval 1 minute per periodontal pocket). The active phase of the treatment was followed with four visits (one per week) for control of the oral hygiene effectiveness and supragingival plaque removal (abrasive aerosol). Full mouth periodontal status evaluation was recorded again on the last visit.

\section{RESULTS:}

The results from this study show significant improvement of the clinical parameters of periodontitis in all treated patients and in almost all periodontal sites. In the regions treated with subgingival irrigation with $10 \%$ povidone-iodine solution superior reduction of the probing depth, more attachment gain and better reduction of the gingival inflammation were recorded in comparison with regions treated with SRP only. The percent of Free from Plaque Surfaces (FPS) is also bigger in the test sites. Concerning level-better results were detected in the test sites.

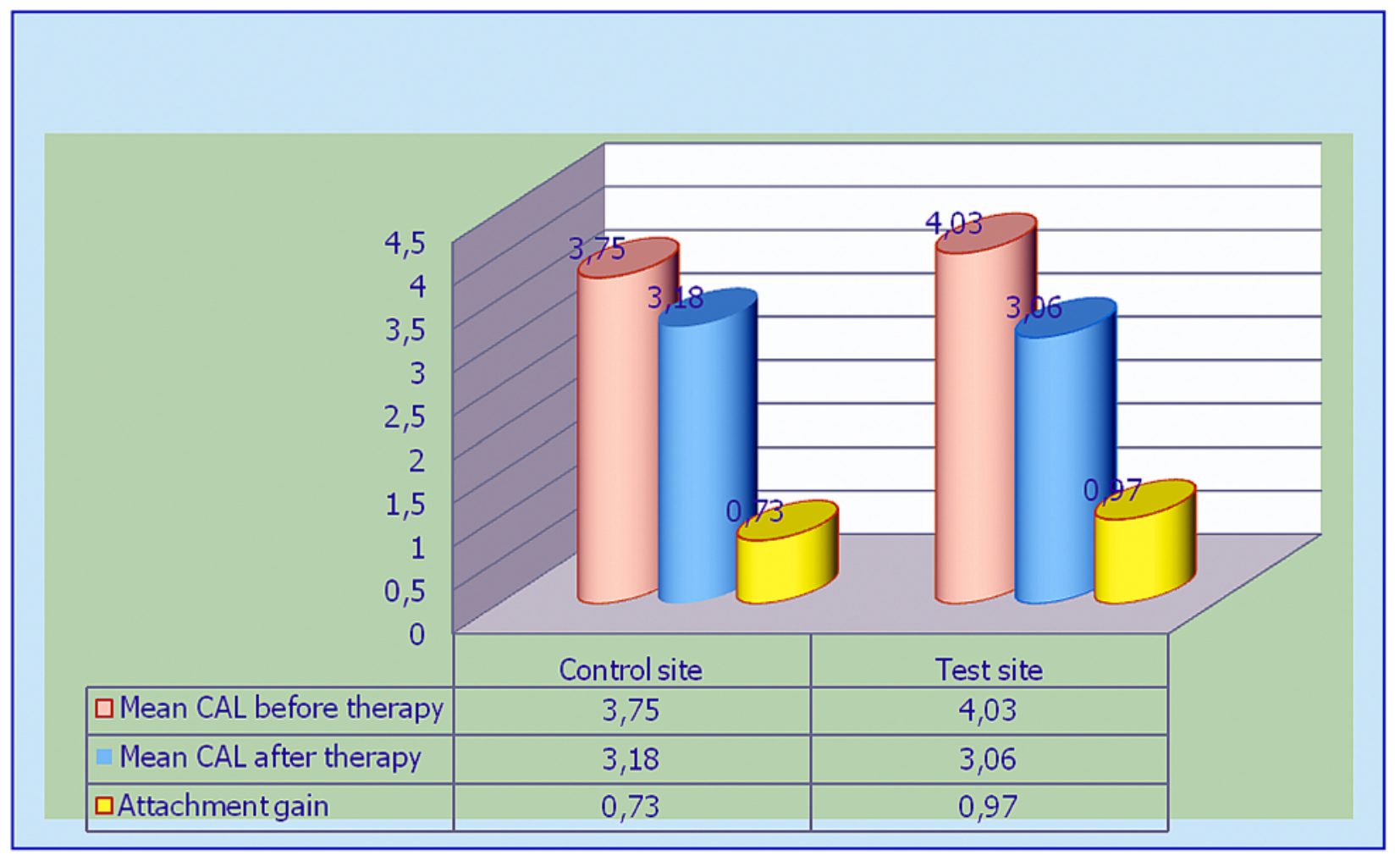

Table 1. Clinical attachment level in $\mathrm{mm}$ 


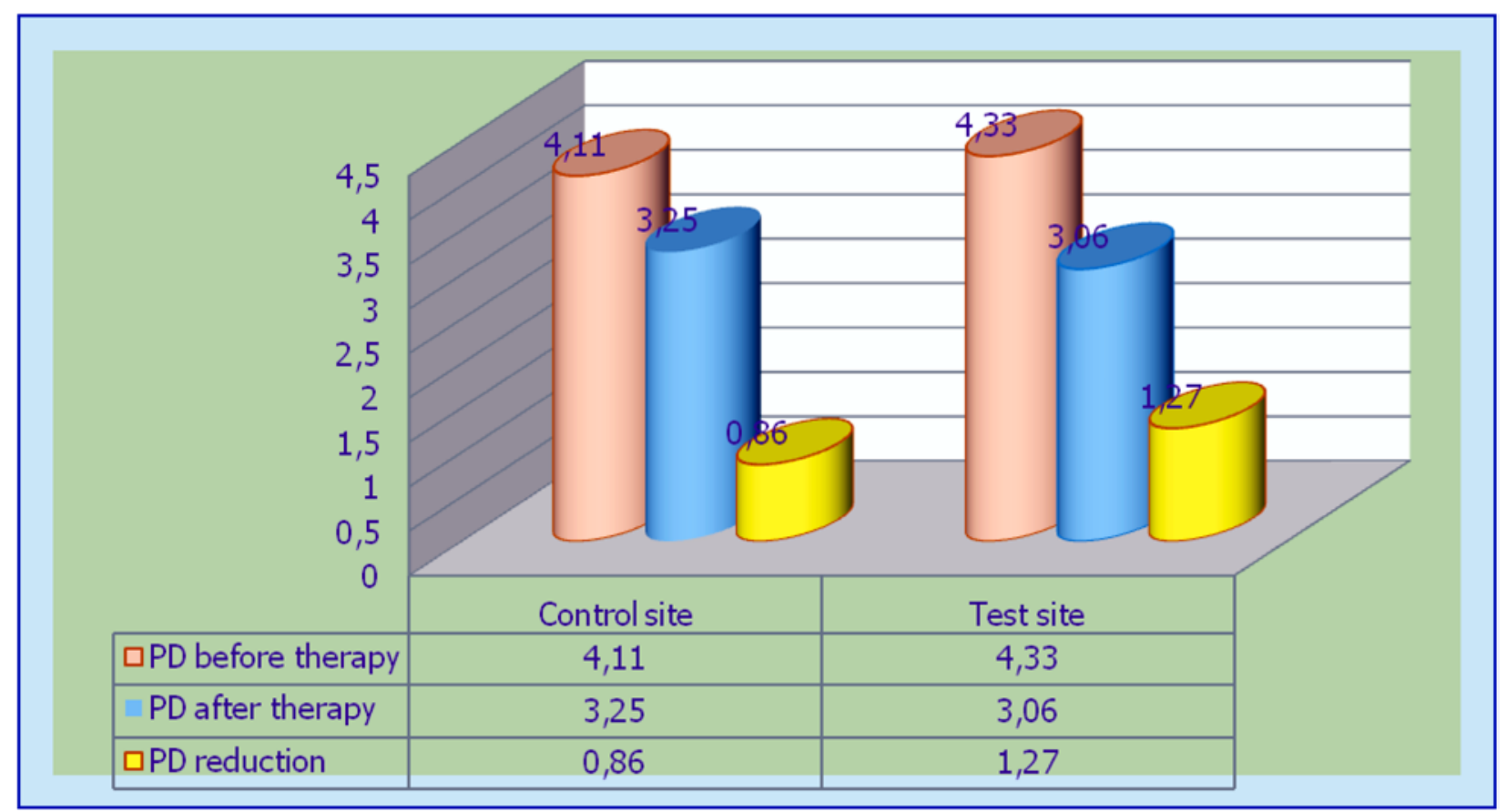

Table 2. Probing depth in $\mathrm{mm}$.

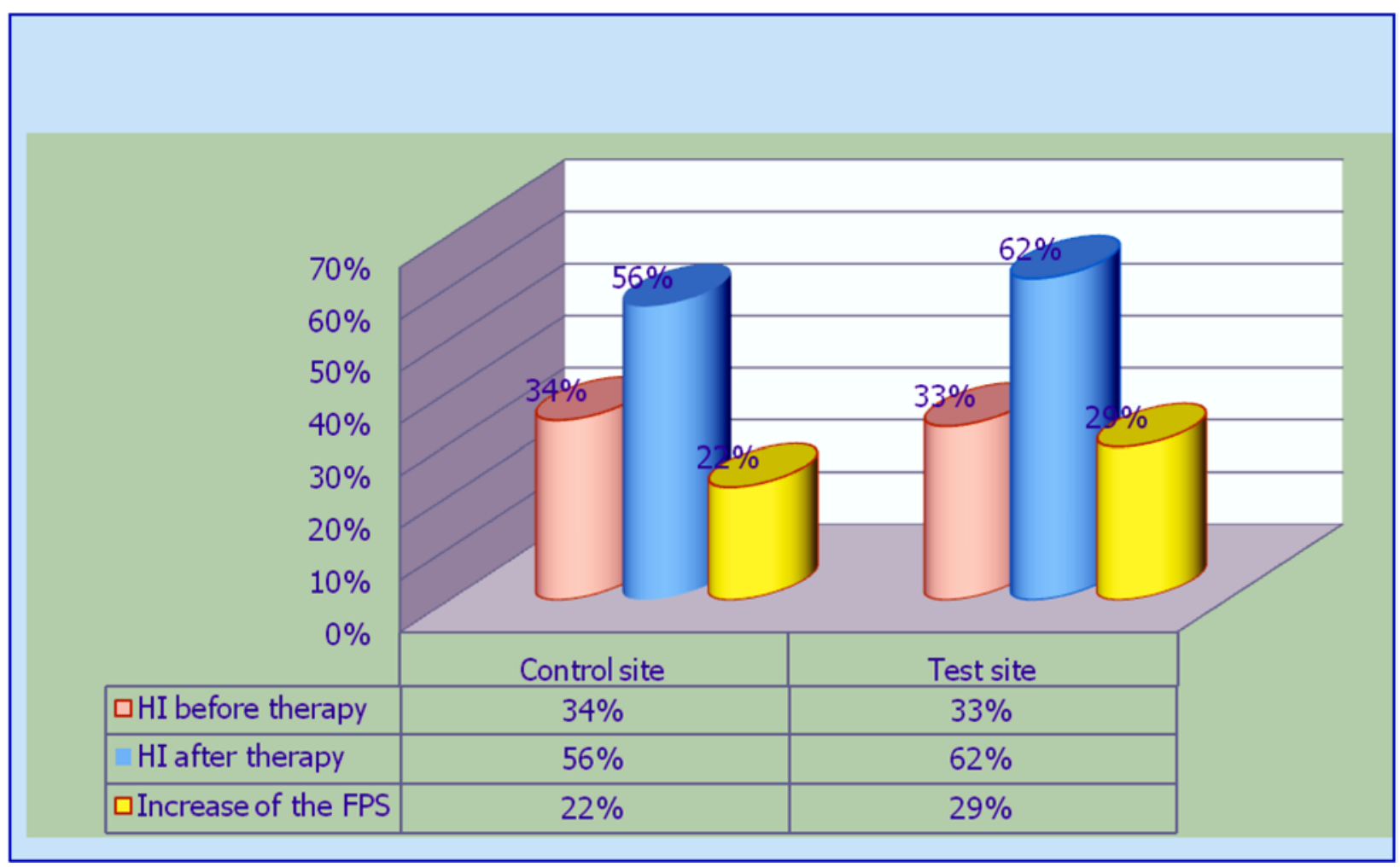

Table 3. Plaque reduction. 


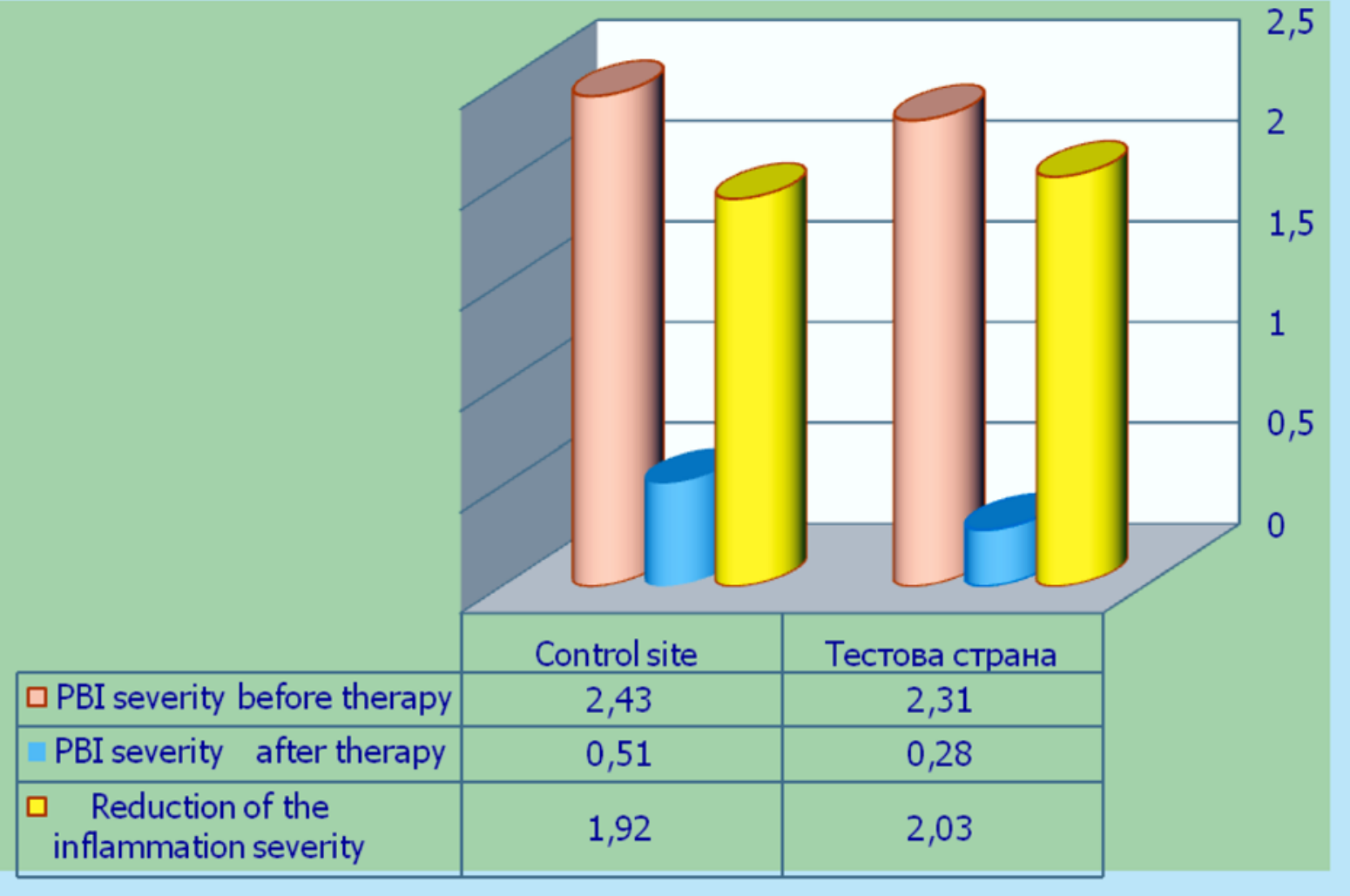

Table 4. Gingival inflammation - severity.

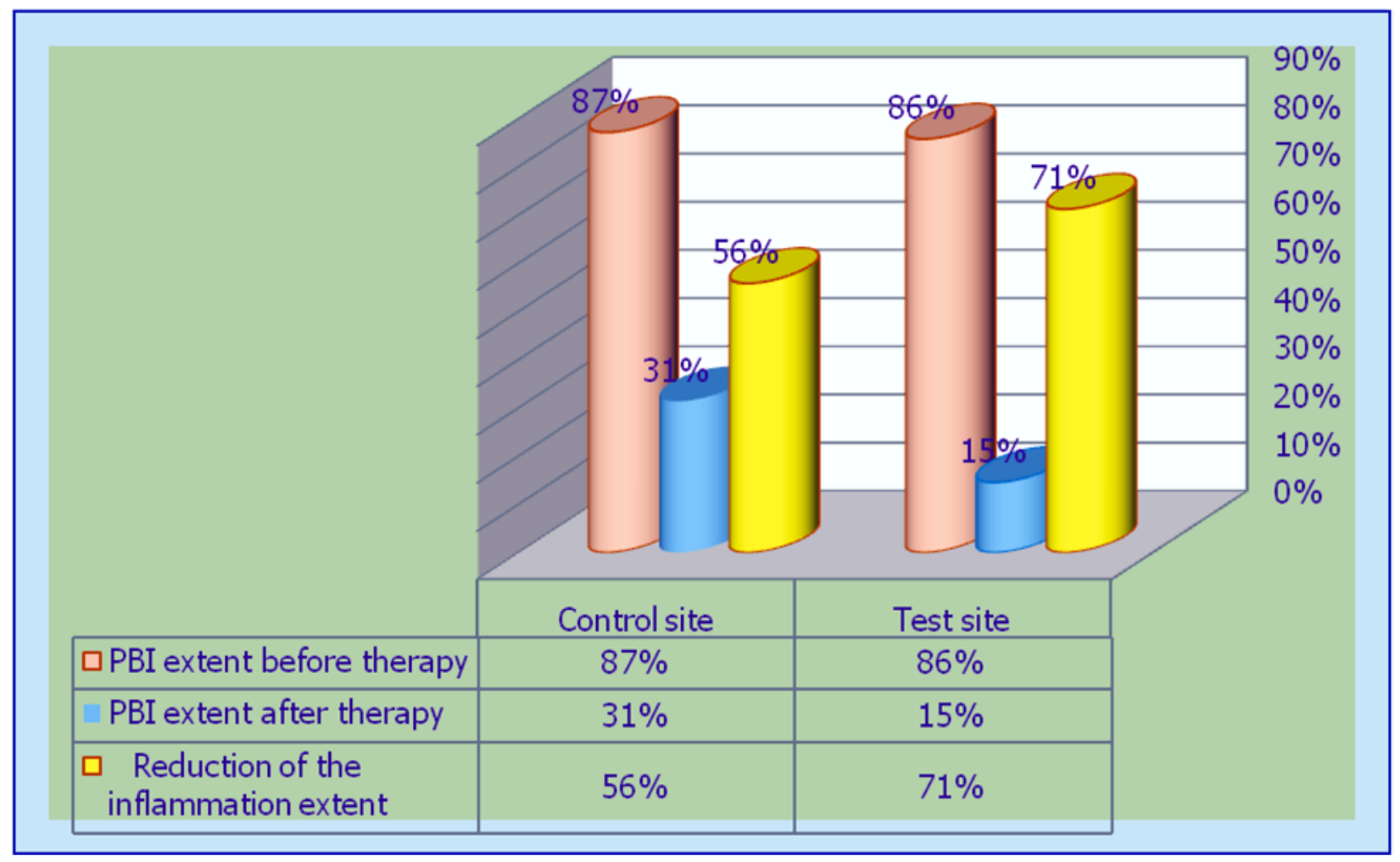

Table 5. Gingival inflammation - extent. 


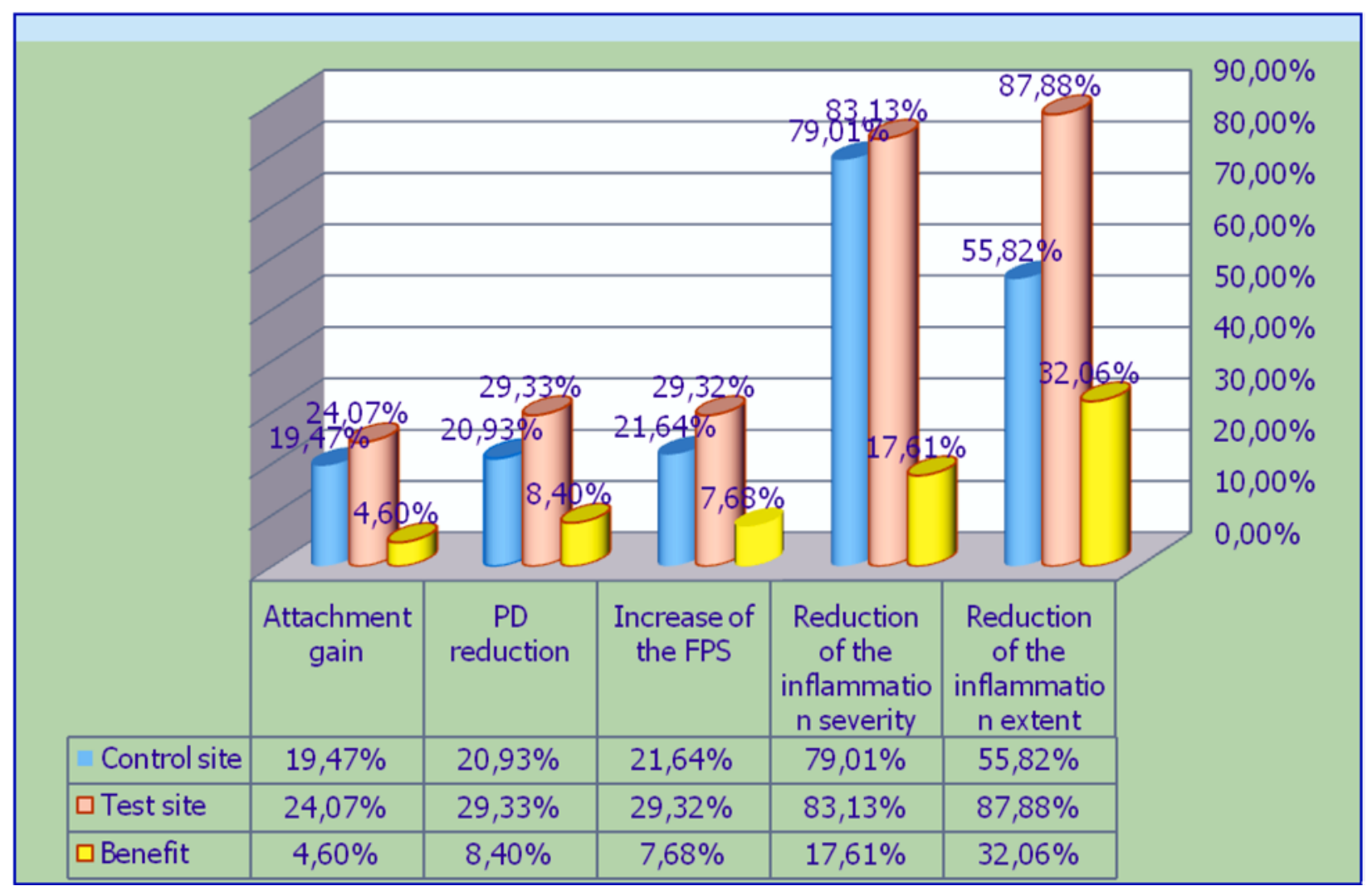

Table 6. RESULTS

\section{CONCLUSION:}

This study demonstrates positive effect of the subgingival irrigations with $10 \%$ povidone-iodine in cases of severe chronic periodontitis. This results are rationale for future studies on the effectiveness of the iodophore solutions in the treatment of the disease and on the clinical significance of the investigated treatment.

\section{REFERENCES:}

1. Burks R1. Povidone-iodine solution in wound treatment. Phys Ther 1998; 78:212-218.

2. Caufield P. W., Allen D. N., Childers N. K. In vitro susceptibilities of suspected parodontopathic anaerobes as determined by membrane transfer assay. Antimicrob Agents Chemother 1987,31:1989-1993.

3. Higashitsutsumi M., Kamoi K., Miyata H. et al. Bactericidal effects of povidone-iodine solution to oral pathogenic bacteria in vitro. Postgrad Med J 1993;69: S10-S14.

4. Kunisada T., Yamada K., Oda S., Hara O. Investigation on the efficacy of povidone-iodine against antiseptic-resistant species. Dermatology 1997; 195 (Suppl.2):
14-18.

5. Modulation of the Host Response in Periodontal Therapy. J Periodontol;73:460470, 2002

6. Muller R.F., Hopfner C., Lange D. E. Efficacy of a PVP-iodine compound on selected pathogens of the oral cavity in vitro (in German). Dtsch Zahnarztl Z 1989;44: 366-369.

7. Numazaki K, Asanuma H. Inhibitory effect of povidone-iodine for the antigen expression of human cytomegalovirus. In Vivo 1999;13:239-241.

8. Quirynen M., Teughels W., De Soete M. and Van Steenberghe D. Topical Antiseptics And Antibiotics In The Initial
Therapy Of Chronic Adult Periodontitis: Microbiological Aspects. Periodontol 2000; 28:72-90, 2002.

9. Shreier H., Erdos G., Reimer K., Konig B., Konig W.,Fleisher W. Molecular effects of povidone-iodine on relevant microorganisms: An electron-microscopic and biochemical study. Dermatology 1997; 195(Suppl.2):111-117.

10. Slots J, Contreras A. Herpesviruses: a unifying causative factor in periodontitis? Oral Microbiol Immunol 2000;15:277-280.

11. Slots J. Selection of antimicrobial agents in periodontal therapy. J Periodont Res 2002; 37; 389-398. 